\title{
PROPERTIES OF Fe, Ni AND Zn ISOTOPE CHAINS NEAR THE DRIP-LINE
}

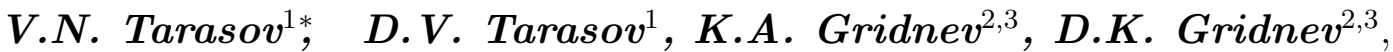 \\ V.G. Kartavenko ${ }^{3,4}$, W. Greiner ${ }^{3}$, V.I. Kuprikov ${ }^{1}$ \\ ${ }^{1}$ National Science Center "Kharkov Institute of Physics and Technology", 61108, Kharkov, Ukraine \\ ${ }^{2}$ Institute of Physics, St. Petersburg State University, Russia \\ ${ }^{3}$ Frankfurt Institute of Advanced Studies J.W.G. University, Frankfurt, Germany \\ ${ }^{4}$ Joint Institute for Nuclear Research, Dubna, Russia
}

(Received April 21, 2007)

\begin{abstract}
The location of proton and neutron drip-lines and the characteristics of the neutron-deficient and the neutron-rich isotopes $\mathrm{Fe}, \mathrm{Ni}$ and $\mathrm{Zn}$ on the basis of Hartree-Fock method with Skyrme forces (Ska, SkM*, Sly4) taking into account deformation was investigated. The calculations predict a big jump of deformation parameter up to $\beta \sim 0.4$ for Ni isotopes in the neighborhood of $N \sim 62$. The manifestation of magic numbers for isotopes ${ }^{48} \mathrm{Ni},{ }^{56} \mathrm{Ni},{ }^{78} \mathrm{Ni}$ and also for the stable isotope in the respect to neutron emission ${ }^{110} \mathrm{Ni}$ which is situated beyond the neutron drip-line is discussed.
\end{abstract}

PACS: 21.60.Jz, 21.10.Dr

1. The structure of nuclei which are very far from the valley of stability and the location of proton and neutron drip-lines are one of the most important tasks of nuclear physics. The nuclei with neutron excess are region of great interest $[1,2,3,4,5]$. However the question about the existence of stability islands of the nuclei with a very big neutron excess is not studied enough up to now. In our previous works $[6,7]$ we presented the results of our investigations in search of very neutron-rich stable nuclei which are far beyond neutron nuclear drip-lines on the basis of Hartree-Fock (HF) method with Skyrme forces accounting deformation (DHF). In particular, for neutron-rich nuclei with $6 \leq Z \leq 16$ in the neighborhood of neutron nuclear drip-line it was predicted the existence of a stability peninsula which rests on the isotope ${ }^{40} \mathrm{O}$.

A lot of attention has been given recently to the study of the properties of the neutron-deficient and the neutron-rich nuclei in the region of $\mathrm{Fe}$ and $\mathrm{Ni}$ $[8,9]$. Doubly magic neutron-deficient isotope ${ }^{48} \mathrm{Ni}$ [10] and doubly magic isotope ${ }^{78} \mathrm{Ni}$ [11] have been experimentally discovered lately.

In the present paper we theoretically investigated the location of proton and neutron drip-lines for Fe, $\mathrm{Ni}$ and $\mathrm{Zn}$ isotopes on the basis of HF method with Skyrme forces accounting deformation.

2. Rather clear and complete description of $\mathrm{HF}$ method one can find in [12]. In our calculations we used the parameterisation of Skyrme forces Sly4 [13], $S k M^{*}$ [14] and Ska [15]. Pairing effects were included in the BCS approximation and only in the space of bounded one-particle states with the pairing constant $G=(19.5 / A)[1 \pm 0.51(N-Z) / A][16]$, where the sign "+" corresponds to the protons but the sign "-" corresponds to the neutrons. The justification of applicability of this approximation was given in $[6,7]$.

We have used the iteration method to solve the system of DHF equations which is described in details in $[7,17,18]$. In this method required oneparticle wave functions DHF are expanded in series of complete set of eigenfunctions of axially deformed harmonic oscillator with the frequencies $\omega_{r}$ and $\omega_{z}$ - The parameters of basis $q=\omega_{r} / \omega_{z}$ and $\beta_{0}=$ $\left[m\left(\omega_{r}^{2} \omega_{z}\right)^{1 / 3} / \hbar\right]^{1 / 2}$ have been chosen on each iteration such that the total energy of nucleus $E=\left(q, \beta_{0}\right)$ was minimal. The optimization of $q$ and $\beta_{0}[7,18]$ on each iteration is important for the calculations of weakly bounded neutron-rich nuclei near the dripline. The densities of neutron distributions of such systems have big root mean squares radii and can have neutron halo $[1,2,3,4,5]$. That's why it is important to describe correctly the asymptotic behavior of wave functions for big $r$. In our calculations the decrease of optimal $\beta_{0}$ with approaching to neutron drip-line is always accompanied by increase of the calculated root mean squares radii. Thus, in our case, the asymptotic behavior of basis wave functions changes depending on space spread density with guaranteeing the highest value of nuclear binding energy.

3. Let's go over the results of our calculations comparing with the data which were obtained on the

*Corresponding author. E-mail address: vtarasov@kipt.kharkov.ua 
basis of Hartree-Fock-Bogoliubov (HFB) method by the group of authors M.V. Stoitsov, J. Dobaczewski, W. Nazarewicz et.al. in [19].

3.1. Ni isotopes. In our calculations for $S k a$ forces the separation energies of one proton $S_{p}$ for neutron-deficient $\mathrm{Ni}$ isotopes remain positive up to ${ }^{48} \mathrm{Ni}$ including. The last isotope $\mathrm{Ni}$ stable in the respect to one proton emission (proton-stable) corresponds to doubly magic neutron-deficient isotope ${ }^{48} \mathrm{Ni}$ which has been experimentally discovered recently [10]. This result differs from the data obtained in [9] where proton instability of isotope ${ }^{48} \mathrm{Ni}$ was predicted, as the proton drip-line defined by means of the condition for chemical potential $\lambda_{p}=0$ does not correspond to experimental data [10]. The proton drip-line defined by means of the condition $S_{p}=0$ does not change if we use $S k M^{*}$ and Sly 4 forces.

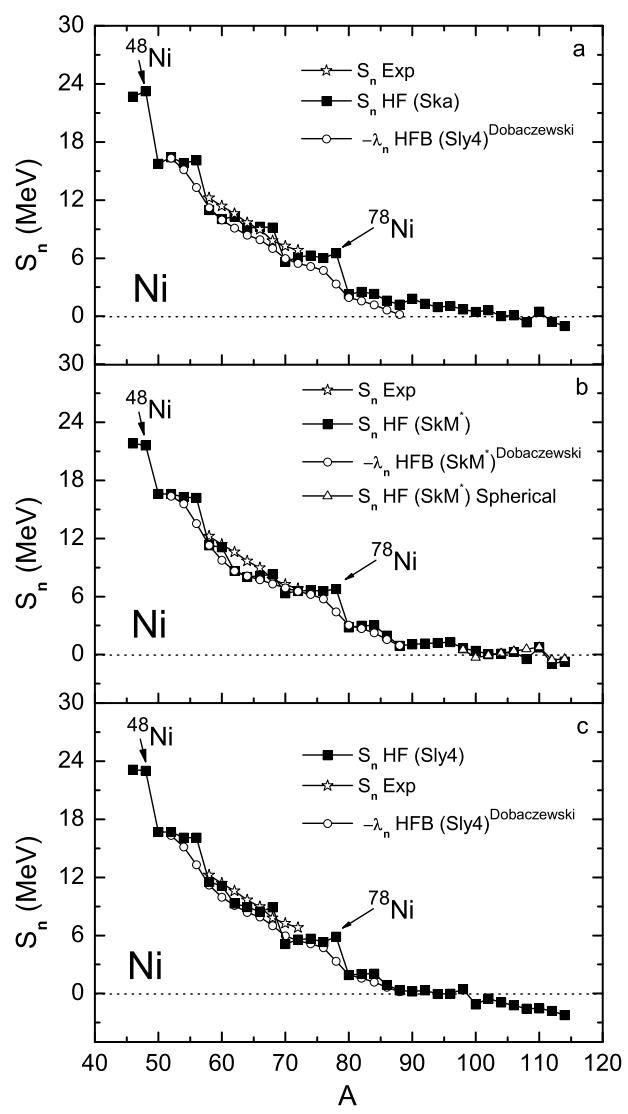

Fig.1. Separation energies of one neutron $S_{n}$ for $N i$ isotopes depending on $A$ compared to the experimental data [20] and to the values of neutron chemical potentials $\lambda_{n}$ (Dobaczewski J.) [19] for forces Ska, SkM* and Sly4. In figure $1 b$ in the vicinity of $A=110$ additionally are shown the data for the spherical $\mathrm{HF}+B C S$ calculations

Fig.1 shows the experimental [20] and calculated separation energies of one neutron $S_{n}$ for different variants of effective forces used in our calculations. It is seen from figure 1 a that the dependence $S_{n}$ from A for $S k a$ forces has specific sharp bends connected with filling of corresponded neutron subshells: specific sharp bend for ${ }^{48} \mathrm{Ni}$ is caused by filling neutron subshell $\nu s_{1 / 2}$, for ${ }^{56} \mathrm{Ni}$ by $\nu f_{7 / 2}$, for ${ }^{68} \mathrm{Ni}$ by $\nu p_{1 / 2}$, for ${ }^{78} \mathrm{Ni}$ by $\nu g_{9 / 2}$. The last isotope with positive value of $S_{n}$ is the isotope ${ }^{106} \mathrm{Ni}$. Isotope ${ }^{108} \mathrm{Ni}$ any more is not the stable isotope with respect to one-neutron emission (neutron-unstable), but the isotope ${ }^{110} \mathrm{Ni}$ again becomes neutron-stable with $S_{n}=0.483 \mathrm{MeV}$. Such increase of stability ${ }^{110} \mathrm{Ni}$ is connected with filling neutron subshell $\nu h_{11 / 2}$. For ${ }^{56} \mathrm{Ni},{ }^{78} \mathrm{Ni}$ and ${ }^{110} \mathrm{Ni}$ increase of value $S_{p}$ is also observed. For these nuclei the corresponding mentioned above neutron subshells and proton subshell $\pi f_{7 / 2}$ are completely filled. In this case the increase of separation energies of neutron and proton can be considered as manifestation of magic numbers $\mathrm{Z}=28, \mathrm{~N}=20,28,40,50,82$. In our calculations it corresponds to zero value of the neutron energy gap $\Delta_{n}$ calculated for mentioned above $G$.

The results presented on figure1b for $S k M^{*}$ forces qualitatively poorly differ from the results for forces $S k a$, showing some stability with respect to a choice of forces, and in particular of increase of stability with respect to emission of neutrons at $A=110\left(S_{n}=0.755\right.$ $\mathrm{MeV})$. In contrast to forces $S k a$, the results obtained for forces $S k M^{*}$ show specific sharp bend in dependence $S_{n}$ on A which corresponds to $\mathrm{N}=32$ and to filling of a subshell $\nu p_{3 / 2}$, and also $\Delta_{n}=0$. Reinforcement of stability of nuclei at $\mathrm{N}=32$ was noticed earlier $[6,7]$ for the isotope ${ }^{40} \mathrm{O}$, and also in work [21]. At the same time for forces $S k M^{*}$ and $\mathrm{N}=40$ the energy gap is $\Delta_{n} \neq 0$. Therefore, for isotopes $\mathrm{Ni}$ manifestation of magic numbers $\mathrm{N}=32,40$ is unstable in relation to a choice of forces.

For forces Sly4 (Fig.1, c) the neutron drip-line corresponds to ${ }^{92} \mathrm{Ni}$ and some splash of stability is observed for ${ }^{98} \mathrm{Ni}$, that corresponds to completely filled subshell $\nu g_{7 / 2}$. We shall note, that force Sly4 always give less bounded decisions and neutron dripline corresponds to smaller A in comparison with the calculations for forces $S k a$ and $S k M^{*}$. For forces $S l y 4$, as well as for forces $S k M^{*}$, in dependence $S_{n}$ on A specific sharp bend corresponding to $\mathrm{N}=32$ is observed. Similarly to proton drip-line, the drip-line in the respect to emission of one neutron, determined by a condition $\lambda_{n}=0$, does not coincide with position of drip-line determined of the condition $S_{n}=0$.

Discussing filling of subshells for of some isotopes $\mathrm{Ni}$, we used classification of states corresponding to spherical symmetry of an average field. It is possible if in addition to make calculations within the limits of method HF [22] directly in coordinate space with spherical symmetry of an average field (SHF). The use of SHF is motivated as, in all the cases we considered, specific sharp bend of value $S_{n}$ corresponds to the decisions obtained within the limits of DHF which have spherical distribution of density.

Let's consider now the character of change for separation energies of two neutrons $S_{2 n}$ depending on A for forces $S k a, S k M^{*}$ and Sly4 in HF calculations and in comparison with HFB [19]. These results are shown on Fig.2. The separation energies of two neutrons is determined as 
$S_{2 n}(Z, N)=E(Z, N)-E(Z, N-2)$, where $E$ is full binding energy of a nucleus. As it is seen in Fig.2, the calculations on the basis of $\mathrm{HF}$ and the calculations HFB approximately with the same quality describe available experimental data for separation energies of two neutrons $S_{2 n}$. As well as for $S_{n}$, the results of calculations $S_{2 n}$ for forces $S k a$ and $S k M^{*}$ show, that these forces provide greater stability of nuclei in the respect to emission of two neutrons, than forces Sly4. The discussed specific sharp bends in dependence $S_{n}$ and $S_{2 n}$ on A are observed at the same $\mathrm{N}$, and their position equally depends on a choice of forces. We cannot calculate separation energy of two neutrons $S_{2 n}$ for ${ }^{110} \mathrm{Ni}$ since the neighbouring isotopes are unstable. As it is seen in Fig.2, the drip-line in the respect to separation of two neutrons is located at smaller values $\mathrm{N}$, than drip-line in the respect to separation of one neutron.

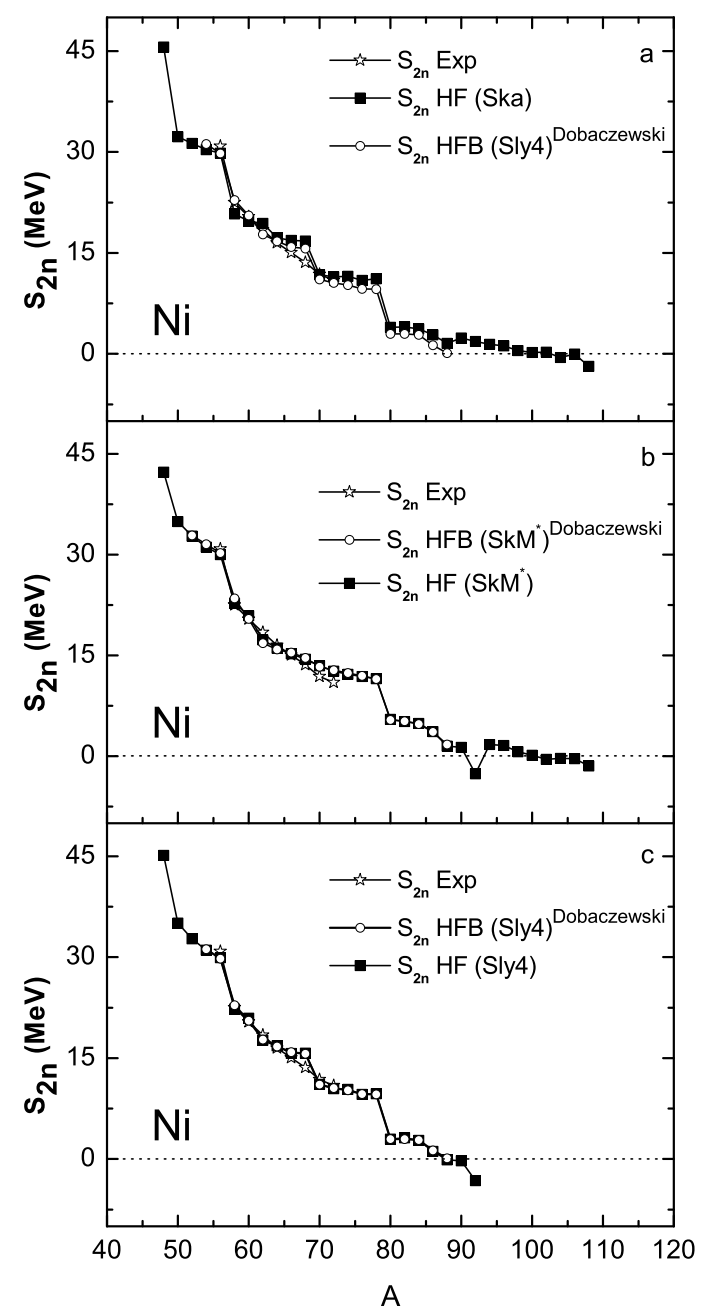

Fig.2. The separation energies of two neutrons $S_{2 n}$ for $N i$ isotopes depending on $A$ compared to the experimental data [20] and the data obtained in HFB calculations (Dobaczewski J.) [19] for forces Ska, SkM* and Sly4

It is rather unexpected that the results for calculations of $S_{2 n}$ by method $\mathrm{HF}$ and HFB completely coincide if the calculations are made with the same types of forces (see Fig.2, b and Fig.2, c). Such good description of separation energies of two neutrons $S_{2 n}$ for the considered region of nuclei in comparison with the method HFB in which pairing is made including continuum, gives the basis, contrary to historically accepted opinion $[2,9]$, to use our version of method $\mathrm{HF}+\mathrm{BCS}$ for research of properties of nuclei near the drip-line. As additional argument in favour of it we can consider the results which we obtained for the root mean square radii $\left\langle r_{n, p}^{2}\right\rangle^{1 / 2}$ which are presented in Fig.3, in comparison with data [19].

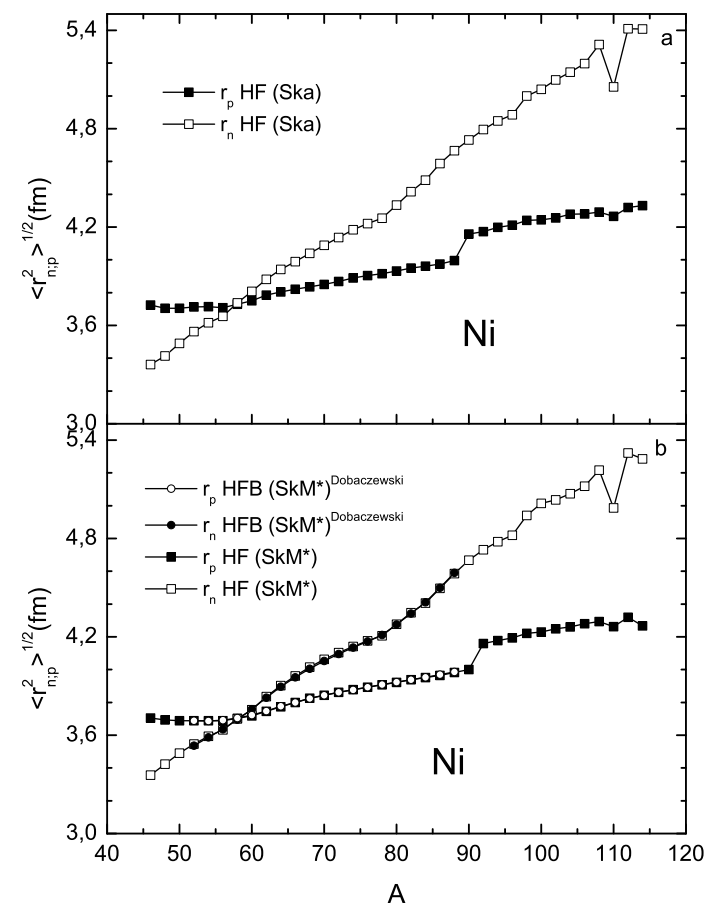

Fig.3. Neutron and proton root mean square radii $\left\langle r_{n, p}^{2}\right\rangle^{1 / 2}$ of $N i$ isotopes depending on $A$ for forces Ska, SkM* and the data obtained in HFB calculations (Dobaczewski J.) [19] for forces SkM*

Just bad description $\left\langle r_{n}^{2}\right\rangle^{1 / 2}$ for isotopes $\mathrm{Ni}$ [2], was in due time the basis for the statement about non-applicability of the HF+BCS method for calculations of properties of extremely neutron-rich nuclei. The wrong asymptotic behavior of basic wave functions of harmonic oscillator [19] was considered as the important reason of non-applicability $\mathrm{HF}+\mathrm{BCS}$ for the description of nuclei close to drip-line. We shall note, that in pairing on the basis of BCS we take into account in our calculations only the bounded one-particle states and consequently influence of continuum explicitly is not considered, and asymptotic behavior of basic wave functions is corrected by the procedure of optimization of oscillator parameters $q$ and $\beta_{0}$ on each iteration. As it is seen in Fig.3, b, the agreement radii $\left\langle r_{n, p}^{2}\right\rangle^{1 / 2}$ with data given in [19] more than good. As we explicitly do not consider influence of continuum, it is possible to assume, that correct asymptotic behavior of density for big value of radii 
$r$ has the defining value for the description of $\left\langle r_{n, p}^{2}\right\rangle^{1 / 2}$ . Data [19] for value radii $\left\langle r_{n, p}^{2}\right\rangle^{1 / 2}$ correspond to stable isotopes in respect to emission of two neutrons. The dip in value radii $\left\langle r_{n, p}^{2}\right\rangle^{1 / 2}$ is connected with the spherical shape of ${ }^{110} \mathrm{Ni}$, and jump in value $\left\langle r_{p}^{2}\right\rangle^{1 / 2}$ in the vicinity $A=90$ is connected with big jump in value of deformation parameter $\beta_{p}$. In process of increase of neutron excess the value of the $\left\langle r_{n}^{2}\right\rangle^{1 / 2}$ exceeds the $\left\langle r_{p}^{2}\right\rangle^{1 / 2}$, and approaching to drip-line, this excess becomes very big. For extremely neutron-rich isotopes $N i$ we can speak about presence of neutron skin.

The data for neutron and proton parameters of quadrupole deformation $\beta_{n, p}$ are presented in Fig.4.

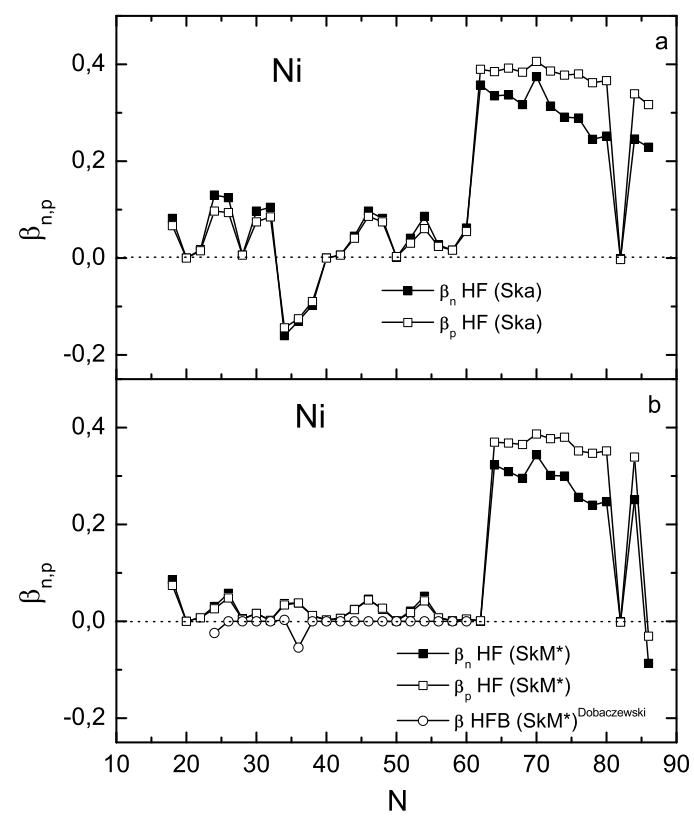

Fig.4. Neutron and proton parameters of quadrupole deformations of isotopes $\mathrm{Ni}$ depending on $A$ for forces Ska, SkM* and the data obtained in HFB calculations (Dobaczewski J.) [19] for forces $S k M^{*}$

For the isotopes $\mathrm{Ni}$ corresponding to magic numbers mentioned above the parameters of deformation $\beta_{n, p}$ have zero value, and at $N \sim 62$ the value of parameter of deformation very rapidly increases up to value $\beta \sim 0.35-0.4$, and $\beta_{p}$ is more than $\beta_{n}$. We also note, that ${ }^{110} \mathrm{Ni}$ has spherical form. Significant increase of deformation near the drip-line is possible not only for isotopes Ni. For example, in work [23] on the basis of method HFB with forces Sly4 for isotopes $\mathrm{Zr}$ near to neutron drip-line there is a very big increase of parameter of quadrupole deformation up to $\beta_{n, p} \sim 0.42 \div 0.47$, that coincides with the experimental data.

3.2 Fe and $\mathrm{Zn}$ isotopes. The investigation of nuclei with $\mathrm{Z}-2$ and $\mathrm{Z}+2$ with the respect to magic nucleon with $\mathrm{Z}=28$ is of certain interest. For isotopes Fe and $\mathrm{Zn}$ all the calculations were made only with forces $S k a$.

The last stable isotope in the respect to emission of one proton, as well as in the calculations [19] is isotope ${ }^{46} \mathrm{Fe}$, that can be considered as manifestation of magic number $\mathrm{N}=20$.

For isotopes $\mathrm{Zn}$ the last stable isotope in the respect to emission of one proton is isotope ${ }^{54} \mathrm{Zn}$. It does not coincide with the data of the work [19] for forces Sly 4 where last the stable isotope in the respect to emission of one proton is the isotope ${ }^{58} \mathrm{Zn}$ if the drip-line is defined by means of chemical potential $\lambda_{p}$.

The separation energies of one neutron $S_{n}$ and results of calculations $\lambda_{p}$ [19] for forces Sly 4 are presented in Fig.5. It is seen, that dependence $S_{n}$ on A for isotopes $\mathrm{Fe}$ and $\mathrm{Zn}$ differs from similar dependence for isotopes $\mathrm{Ni}$. The shell effects which are manifested in the form of specific sharp bend for isotopes $\mathrm{Ni}$ are poorly expressed for isotopes $\mathrm{Fe}$.

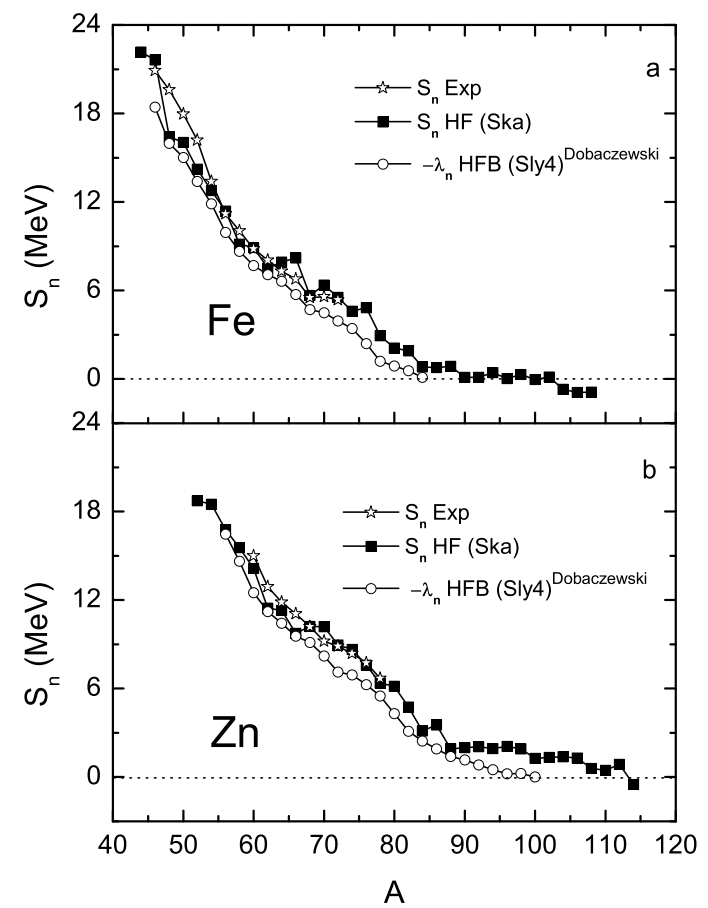

Fig.5. Separation energies of one neutron $S_{n}$ for isotopes $F e$ and $Z n$ for forces Ska depending on $A$ compared to the experimental data [20] and values of neutron chemical potentials (Dobaczewski J.) [19] for forces Sly4

It can be partially explained if to admit, that there is no reciprocal gain of proton and neutron magic numbers for isotopes Fe. For isotopes $\mathrm{Fe} \mathrm{Z}=26$ is two units less than the magic number 28 and proton subshell $1 f_{7 / 2}$ is not filled. From $A=90$ up to $A=98$ the value $S_{n}>0$, but the value of it is very small. The last neutron-rich stable isotope is ${ }^{98} \mathrm{Fe}$ and the stable isotope ${ }^{102} \mathrm{Fe}$ has $S_{n}=0.146 \mathrm{MeV}$.

For isotopes $\mathrm{Zn} \mathrm{Z}=30$ is two units more than the magic number 28 and the proton subshell $1 f_{7 / 2}$ is filled, and the next proton subshell $2 p_{3 / 2}$ is halffilled. The neutron-rich isotopes $\mathrm{Zn}$ are more stable than the isotopes $\mathrm{Fe}$ in the respect to emission of 
one neutron. The last neutron-rich stable isotope is ${ }^{112} \mathrm{Zn}$ that corresponds to filling of a neutron subshell $\nu h_{11 / 2}$ as in the stable isotope ${ }^{110} \mathrm{Ni}$. The separation energy of one neutron for ${ }^{112} \mathrm{Zn}$ in DHF calculation is $S_{n}=0.87 \mathrm{MeV}$.

\section{THE CONCLUSION}

The research made in the present work for the chains of isotopes $\mathrm{Fe}, \mathrm{Ni}$ and $\mathrm{Zn}$ on the basis of Hartree-Fock method with Skyrme forces accounting deformation allows to extract the following results:

- for $\mathrm{Ni}$ isotopes the last stable isotope in the respect to emission of one proton is doubly magic isotope ${ }^{48} \mathrm{Ni}$ that was proved experimentally [10];

- beyond the neutron drip-line of nickel isotopes, coinciding with ${ }^{106} \mathrm{Ni}$ our calculations predict the existence of neutron-rich isotope ${ }^{110} \mathrm{Ni}$ that can be to considered as manifestation of magic number $\mathrm{N}=82$;

- in our calculations for isotopes $\mathrm{Ni}$ it was obtained good agreement of $S_{2 n}$ and $\left\langle r_{n, p}^{2}\right\rangle^{1 / 2}$ with the data of calculations based on the method HFB that gives the basis for application of the method $\mathrm{HF}+\mathrm{BCS}$ for studying extremely neutron-rich isotopes;

- for $\mathrm{Ni}$ isotopes approaching to drip-line and at $\mathrm{N}=60$ the value of deformation parameter jumps up to $\beta_{n, p} \sim 0.35-0.4$ and the isotope ${ }^{110} \mathrm{Ni}$ has spherical form;

- for isotopes Fe the last stable isotope in the respect to emission of one proton is the isotope ${ }^{46} \mathrm{Fe}$, and for isotopes $\mathrm{Zn}-{ }^{54} \mathrm{Zn}$;

- for isotopes Zn last stable in the respect to emission of one neutron is ${ }^{112} \mathrm{Zn}$; it corresponds to filling of neutron subshell $\nu h_{11 / 2}$, as well as in a stable isotope ${ }^{110} \mathrm{Ni}$.

- the obtained results show that the structure of the drip-line can be rather complex and it is connected with the manifestation of shell structure.

\section{REFERENCES}

1. B. Jonson. Light dripline nuclei // Phys. Rep. 2004, v.389, p. 1-59.

2. J.Dobaczewski, W.Nazarewicz, T.R.Werner. Neutron radii and skins in the Hartree-FockBogoliubov calculations // Z. Phys. 1996, A354, p.27-35.

3. S. Mizutori, J. Dobaczewski, G. A. Lalazissis, W. Nazarewicz, and P.-G. Reinhard. Nuclear skins and halos in the mean-field theory //Phys. Rev. 2000, C61, 044326, p.14.
4. K. Bennaceur, J. Dobaczewski, M. Ploszajczak. Pairing anti-halo effect //Phys. Lett. 2000, B496, p.154-160.

5. J. Meng, H. Toki, S. G. Zhou, S. Q. Zhang, W. H. Long and L. S. Geng. Relativistic Continuum Hartree Bogoliubov Theory for Ground State Properties of Exotic Nuclei: Preprint. arXiv:nucl-th/0508020, 2005, p.79.

6. K. A. Gridnev, D.K. Gridnev, V.G. Kartavenko, V.E. Mitroshin, V.N. Tarasov, D.V. Tarasov, W. Greiner. Specific features of the nuclear dripline in the region of light nuclei // Phys. Atom. Nucl. 2006, v.69, p.1-8.

7. K. A. Gridnev, D.K. Gridnev, V.G. Kartavenko, V.E. Mitroshin, V.N. Tarasov, D.V. Tarasov, W. Greiner. On stability of the neutron-rich oxygen isotopes // Int. Jour. Mod. Phys. 2006, E15, p.673-683.

8. A. F. Lisetskiy, B. A. Brown, M. Horoi, and H. Grawe, A.F. New $T=1$ effective interactions for the $f 5 / 2$, p3/2 p1/2 g9/2 model space: Implications for valence-mirror symmetry and seniority isomers // Phys. Rev. 2004, C70, 044314, p.5.

9. W. Nazarewicz, J. J. Dobaczewski, T.R. Werner, J.A. Maruhn et al. Structure of proton drip-line nuclei around doubly magic ${ }^{48} \mathrm{Ni} / /$ Phys. Rev. 1996, C53, p.470-751.

10. B. Blank, M. Chartier, S. Czajkowski, J. Giovinazzo et al. Discovery of doubly magic ${ }^{48} \mathrm{Ni} / /$ Phys. Rev. Lett. 2000, v.84, p.1116-1119.

11. P. T. Hosmer, H. Schatz, A. Aprahamian, O. Arndt et al. Half-life of the doubly magic rprocess nucleus ${ }^{78} \mathrm{Ni} / /$ Phys. Rev. Lett. 2005, v.94, 112501. p.4.

12. M. Bender, P.-H. Heenen, P.-G. Reinhard. Selfconsistent mean-field models for nuclear structure // Rev. Mod. Phys. 2003, v.75, p.121-180.

13. E. Chabanat, P. Bonche, P. Haensel, J. Meyer, R. Schaeffer. A Skyrme parametrization from subnuclear to neutron star densities Part II. Nuclei far from stabilities // Nucl. Phys. 1998, A635, p.231-256; Nucl. Phys. 1998, A643, p.441442.

14. J. Bartel, P. Quentin, M. Brack et al. Towards a better parametrisation of Skyrme-like effective forces: A critical study of SkM force // Nucl. Phys. 1982, A386, p.79-100.

15. H.S Köhler. Skyrme force and mass formula // Nucl. Phys. 1976, A258, p.301-316.

16. V.E. Mitroshin. Dynamical collective model: even-even nuclei // Yad. Fiz. 2005, v.68, p.13681406 (in Russian). 
17. D. Vautherin. Hartree-Fock calculatins with Skyrme's interactin. II. Axiali deformed nuclei // Phys.Rev. 1973, C7, p.296-316.

18. V.N. Tarasov, V.Yu. Gonchar, Yu.V. Kirichenko, E.V. Inopin. Investigation of properties of deformed nuclei on the base of Hartree-Fock method with effective interaction of Skyrme's type: Preprint KFTI 85-32. Moscow: Atominform, 1985, $13 \mathrm{p}$.

19. M.V. Stoitsov, J. Dobaczewski, W. Nazarewich, S. Pittel, D.J. Dean. Systematic study of deformed nuclei at the drip lines and beyond // Phys. Rev. 2003, C68, 054312, p.11.; http://www.fuw.edu.pl/ dobaczew/thodri/thodri. html
20. G. Audi, A.H.Wapstra, C.Thibault. The AME2003 atomic mass evaluation (II) // Nucl. Phys. 2003, A729, p.337-676.

21. J. Dobaczewski. Tensor interactions in meanfield approach: Preprint: arXiv:nucl-th/0604043, 2006, p.5.

22. E.V. Inopin, V.Yu. Gonchar, V.I. Kuprikov. Charge density changes of nuclei at addition of neutron // Yad. Fiz. 1976, v.24, p.40-43 (in Russian).

23. A. Blazkiewicz, V. E. Oberacker, A. S. Umar, M. Stoitsov. Coordinate space HFB calculations for the zirconium isotope chain up to the two-neutron drip-line: Preprint. arXiv:nuclth/0502063, 2005, p.9.

\title{
СВОЙСТВА ЦЕПОЧЕК ИЗОТОПОВ Fе, Ni И Zn ВБЛИЗИ ГРАНИЦЫ СТАБИЛЬНОСТИ
}

\author{
В.Н. Тарасов, Д.В. Тарасов, К.А. Гриднев, Д.К. Гриднев, \\ В.Г. Картавенко, В. Грайнер, В.И. Куприков
}

На основе метода Хартри-Фока с силами Скирма (Ska, SkM*, Sly4) при учете деформации исследовано положение протонной и нейтронной границы стабильности и характеристики нейтронодефицитных и нейтроноизбыточных изотопов $\mathrm{Fe}, \mathrm{Ni}$ и $\mathrm{Zn}$. Расчеты предсказывают, что для изотопов $\mathrm{Ni}$ в окрестности $N \sim 62$ наблюдается большой скачок величины параметра деформации до $\beta \sim 0.4$. Обсуждается проявление магических чисел для изотопов никеля ${ }^{48} \mathrm{Ni},{ }^{56} \mathrm{Ni},{ }^{78} \mathrm{Ni}$, а также для нейтроностабильного изотопа ${ }^{110} \mathrm{Ni}$, который находится за пределами границы стабильности.

\section{ВЛАСТИВОСТІ ЛАНЦЮЖКІВ ІЗОТОПІВ Fe, Ni I Zn ПОБЛИЗУ ГРАНИЦІ СТАБІЛЬНОСТІ}

\section{В.М. Тарасов, Д.В. Тарасов, К.А. Гріднев, Д.К. Гріднєв, В.Г. Картавенко, В. Грайнер, В.І. Купріков}

На основі метода Хартрі-Фока з силами Скірма (Ska, SkM*, Sly4) при врахуванні деформації досліджено положення протонної і нейтронної границі стабільності і характеристики нейтронодефіцітних і нейтрононадлишкових ізотопів $\mathrm{Fe}, \mathrm{Ni}$ и $\mathrm{Zn}$. Розрахунки завбачають, що для ізотопів $\mathrm{Ni}$ в околі $N \sim 62$ спостерігається великий стрибок величини параметра деформації до $\beta \sim 0.4$. Обговорюються прояви магічних чисел для ізотопів нікелю ${ }^{48} \mathrm{Ni},{ }^{56} \mathrm{Ni},{ }^{78} \mathrm{Ni}$, а також для нейтроностабільного ізотопу ${ }^{110} \mathrm{Ni}$, який знаходиться за межами границі стабільності. 